\title{
Vaccine program rubs out rubella in the US
}

he remarkable contribution of vaccination programs to public health cannot be contested. The success of Jonas Salk's polio vaccine in mass human trials, the fif-

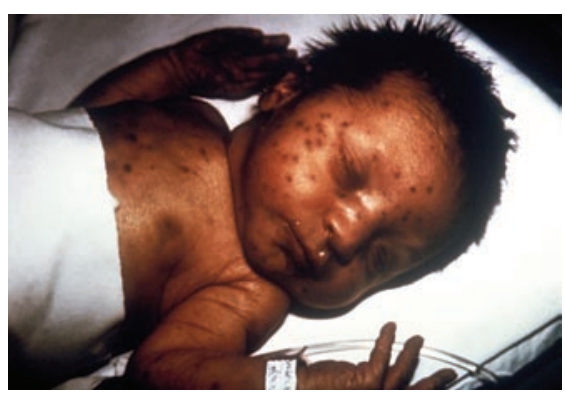

Infant with "blueberry muffin" skin lesions indicative of rubella, a major cause of serious birth defects, which has been eliminated in the US. Photo courtesy of the Centers for Disease Control. tieth anniversary of which was celebrated on April 12, 2005, was one of the most important feats in the history of medicine. As a result of this prevention strategy, the devastating epidemics that plagued the country in the twentieth century have not since occurred, and the World Health Organization declared the US polio-free in 1994. With this announcement, polio was added to the list of diseases that have been stamped out through successful, widespread vaccination programs: the global elimination of smallpox was declared in 1979 , and in 2000 , measles was pronounced no longer endemic in the US.

Rubella (also known as German measles) followed suit on March 21, 2005, when the Centers for Disease Control (CDC) declared it eliminated in the US. The rubella virus was once a grave health threat to infants and a major cause of serious birth defects and miscarriages. The CDC embarked on a rubella elimination program in 1989 , and in the following decade, only 117 cases were reported. In 2001, for the first time, fewer than 100 US cases were reported, and in 2004, fewer than 10 cases surfaced, all of which were likely acquired in other countries and imported into the US.

Despite extraordinary progress in relegating vaccine-preventable diseases, such diseases endure, predominantly in developing countries. Vaccinations against rubella will continue vigilantly in the US because international travel to areas without steadfast vaccine programs makes it possible for surreptitious cases to enter the country and transmit the disease to vulnerable individuals. The Pan-American Health Organization has set a goal to eliminate rubella from all of North and South America by 2010 .

\section{Stacie Bloom}

\section{Belgian scientists awarded top honors}

$T_{\text {he }}$ prize awarded in Belgium. On May 12, 2005, Désiré Collen of the Flanders Interuniversity Institute for Biotechnology and Peter Carmeliet of the University of Leuven will be honored with this award for their pioneering work in genetics and cardiovascular disease. The JCI spoke with the scientists about the prize and the work leading up to it.

JCI: How were you selected for this prize?

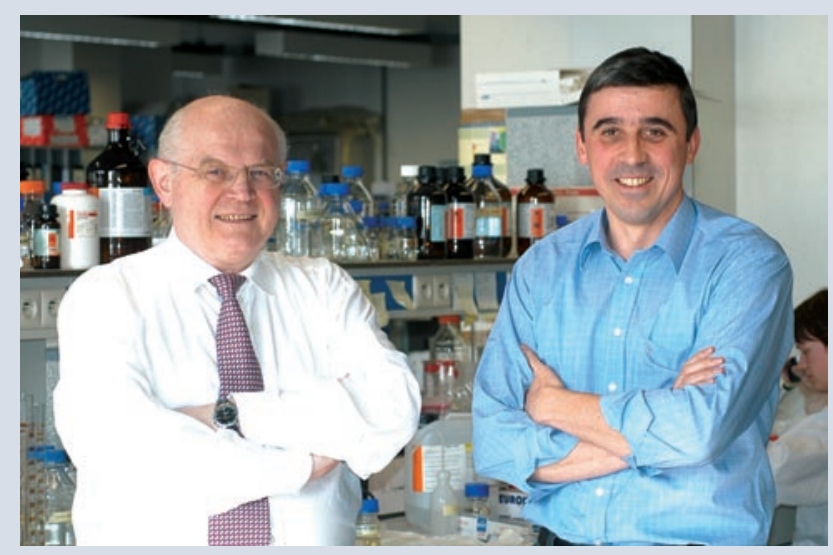

Désiré Collen (left) and Peter Carmeliet share the Interbrew-Baillet Latour Health Prize for their work in genetics and cardiovascular diseases.
Carmeliet: An international jury of renowned scientists selected the laureates among 21 candidates from 13 different countries.

JCI: What aspect of your work directly contributed to your winning this prize?

Collen: I started my scientific career studying the molecular mechanisms of blood clot dissolution. These studies ultimately resulted in the isolation and characterization of tissue-type plasminogen activator (tPA), which digests blood clots. This led to the development, with Genentech Inc., of recombinant tPA for the treatment of acute myocardial infarction.

Carmeliet: In the early 90s, I teamed up with Désiré Collen to study cardiovascular diseases using gene targeting and focused on the molecular basis of angiogenesis in health and disease. These studies elucidated the role of angiogenic factors, in particular of vascular endothelial growth factor (VEGF) and placental growth factor (PlGF), and tested novel strategies to treat cancer, inflammation, and ischemic tissue disease. Recently, our team provided evidence for the therapeutic potential of VEGF for the neurodegenerative disorder amyotrophic lateral sclerosis (ALS).

JCI: What are the most direct clinical consequences of your work?

Collen: The discovery of tPA and its development as a novel thrombolytic drug has saved the lives of a few percent of the more than 5 million treated patients with myocardial ischemia worldwide.

Carmeliet: Mice deficient in a single VEGF allele revealed the key role of VEGF in angiogenesis. Further studies demonstrated 
that PlGF, a homolog of VEGF, affects angiogenesis in disease but not in health. The implication of these findings is that blocking PlGF might combine efficacy in inhibiting tumor growth with safety of only affecting tumor but not quiescent vessels. Conversely, delivery of PlGF stimulated revascularization of ischemic tissues. Our studies also showed that low levels of VEGF cause motor neuron degeneration, reminiscent of ALS. We have now demonstrated that VEGF prolongs survival in ALS rodent models. Clinical trials are underway.

$J C I$ : What is the biggest challenge you face?

Collen: To secure a steady flow of funding and talented researchers to compete at the front line of basic research and to efficiently transfer technology for potential medical applications to interested industrial partners.

Carmeliet: Understanding the molecular basis of angiogenesis and lymphangiogenesis, using mouse, zebrafish, and frog genetic models. Our focus is evolving from identifying which signals stimulate endothelial cells to migrate and divide, to understanding how blood vessels, lymph vessels, and neurons navigate to their targets. Our discovery that VEGF has neurotrophic effects in animal models of ALS may indicate a new approach to treatment of this and other lifethreatening neurodegenerative disorders. A challenge will be to initiate clinical trials, evaluating the therapeutic potential of VEGF for ALS.

JCI: In a typical day, how much of your time do you spend in the lab involved in research?

Collen: I am primarily involved in the overall coordination of the research programs of approximately 160 investigators and technical staff. Much of my time is devoted to drug development programs spun out of the laboratory into dedicated spin-offs. I primarily focus on drug development and technology transfer issues.

Carmeliet: Apart from some teaching and administrative duties, I, together with 4 other senior associates, spend my entire time supervising and coaching a research team of 55 postdocs, PhD students, and technicians. I am primarily involved in the conception of projects, writing grants and papers, and managing and coordinating the mouse, zebrafish, and frog facilities.

JCI: What do you think is the most important discovery you have ever made?

Collen: The molecular regulation of physiological fibrinolysis (breaking down blood clots) and its application to fibrin-relative thrombolytic therapy of acute myocardial infarction.

Carmeliet: Three discoveries seem most relevant: the critical role of VEGF in vascular development and disorders; the finding that PlGF is involved in angiogenesis in disease but not in health, and that PIGF stimulates revascularization of ischemic tissues, while PlGF blockers inhibit pathological angiogenesis; the finding that VEGF is a key survival factor for motor neurons in ALS and that VEGF prolongs survival of ALS rodents.

$J C I$ : What is your ultimate scientific goal?

Collen and Carmeliet: Understanding the molecular mechanisms governing cardiovascular and nervous system function, and the development of mechanism-based novel treatments for life-threatening cardiovascular and neurological diseases.

\section{Stacie Bloom}

\section{In the debate of sex and science, Summers, Hopkins, and the X chromosome battle it out}

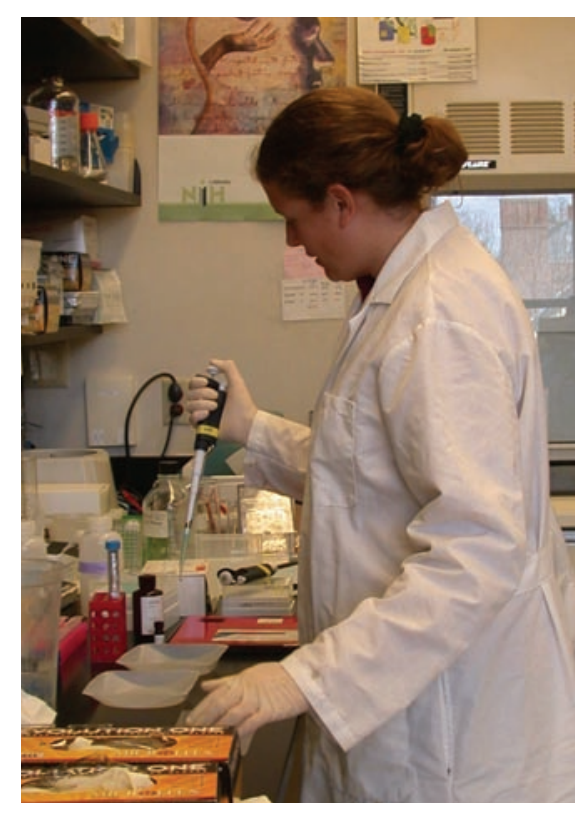

W ence on January 14,2005, Lawrence Summers, the president of Harvard University, suggested that innate biological differences between men and women might be one reason for the paucity of women math and science professors. Nancy Hopkins, a biology professor at the Massachusetts Institute of Technology (MIT), walked out upon hearing this remark. Both Summers' comments and Hopkins' reaction have received significant publicity and undergone much scrutiny.

Hopkins is an accomplished molecular biologist, but it is her pioneering role in

Women are still underrepresented in science, but are unintentional gender biases or innate biological differences to blame? Photo courtesy of Kate Prybylowski (NIH, Bethesda, Maryland, USA). fostering gender equity in academia for which many have come to know her. At the start of her career, Hopkins assumed that so few women worked in science because they wanted families and thus opted out of the 80-hour work week she associated with a successful career in the lab. Hopkins herself married young, but was divorced by 30 and decided not to have children. Early on, Hopkins repeatedly observed men and women equally accomplished in the lab, but noticed that they were not treated the same by colleagues. She did not see women as chairmen or as speakers, nor did she see women faculty with administrative power.

Hopkins says she was in denial about the situation and just worked harder until one particular event, 20 years into her career, opened her eyes. When she needed 